\title{
Research Article \\ On the Modified Jump Problem for the Laplace Equation in the Exterior of Cracks in a Plane
}

\author{
P. A. Krutitskiii ${ }^{1}$ and A. Sasamoto ${ }^{2}$ \\ ${ }^{1}$ KIAM, Miusskaya Sq. 4, Moscow 125047, Russia \\ ${ }^{2}$ AIST, 1-2-1 Namiki, Tsukuba, Ibaraki 305-8564, Japan \\ Correspondence should be addressed to P. A. Krutitskii, krutitsk@mail.ru
}

Received 20 March 2012; Revised 29 March 2012; Accepted 14 April 2012

Academic Editor: Vladimir Mityushev

Copyright (C) 2012 P. A. Krutitskii and A. Sasamoto. This is an open access article distributed under the Creative Commons Attribution License, which permits unrestricted use, distribution, and reproduction in any medium, provided the original work is properly cited.

The boundary value problem for the Laplace equation outside several cracks in a plane is studied. The jump of the solution of the Laplace equation and the boundary condition containing the jump of its normal derivative are specified on the cracks. The problem has unique classical solution under certain conditions. The new integral representation for the unique solution of this problem is obtained. The problem is reduced to the uniquely solvable Fredholm equation of the second kind and index zero. The integral representation and integral equation are essentially simpler than those derived for this problem earlier. The singularities at the ends of the cracks are investigated.

\section{Introduction}

The Dirichlet and Neumann problems outside cracks in a plane for Laplace and Helmholtz equations were treated in [1-10]. The jump problem for the Laplace equation outside cracks in a plane has been studied in [11]. Two boundary conditions of jump type, namely, the jump of the unknown function and the jump of its normal derivative were specified at the cracks in [11]. The so-called modified jump problem has been studied in [12]. In this problem the jump of the normal derivative of the unknown function was not given exactly, but was expressed through limit values of this function on the cracks. The problem in [12] was reduced to the uniquely solvable Fredholm integral equation and the integral representation for a solution of the problem was obtained. However, both integral representation and integral equation derived in [12] were rather complicated. In the present paper we reduce the problem to the simpler integral equation and derive new integral representation for the solution of the problem. The explicit formulas for singularities of the solution gradient at the ends of cracks are presented. 


\section{Formulation of the Problem}

By a simple open curve we mean a nonclosed smooth arc of finite length without self-intersections [10]. In the plane $x=\left(x_{1}, x_{2}\right) \in R^{2}$ we consider simple open curves $\Gamma_{1}, \ldots, \Gamma_{N} \in C^{2, \lambda}$, $\lambda \in(0,1]$, so that they do not have common points. We put $\Gamma=\bigcup_{n=1}^{N} \Gamma_{n}$. We assume that each curve $\Gamma_{n}$ is parametrized by the are length $s$ :

$$
\Gamma_{n}=\left\{x: x=x(s)=\left(x_{1}(s), x_{2}(s)\right), s \in\left[a_{n}, b_{n}\right]\right\}, \quad n=1, \ldots, N,
$$

so that $a_{1}<b_{1}<\cdots<a_{N}<b_{N}$. Therefore points $x \in \Gamma$ and values of the parameter $s$ are in one-to-one correspondence. Below the set of the intervals on the $O s$ axis $\bigcup_{n=1}^{N}\left[a_{n}, b_{n}\right]$ will be denoted by $\Gamma$ also.

The tangent vector to $\Gamma$ at the point $x(s)$ we denote by $\tau_{x}=(\cos \alpha(s), \sin \alpha(s))$, where $\cos \alpha(s)=x_{1}^{\prime}(s), \sin \alpha(s)=x_{2}^{\prime}(s)$. Let $\mathbf{n}_{x}=(\sin \alpha(s),-\cos \alpha(s))$ be a normal vector to $\Gamma$ at $x(s)$. The direction of $\mathbf{n}_{x}$ is chosen such that it will coincide with the direction of $\tau_{x}$ if $\mathbf{n}_{x}$ is rotated anticlockwise through an angle of $\pi / 2$. We consider $\Gamma$ as a set of cracks. The side of $\Gamma$ which is on the left when the parameter $s$ increases will be denoted by $\Gamma^{+}$and the opposite side will be denoted by $\Gamma^{-}$.

We say that the function $u(x)$ belongs to the smoothness class $\mathbf{K}$ if the following conditions are satisfied:

(1) $u(x) \in C^{0}\left(\overline{R^{2} \backslash \Gamma}\right) \cap C^{2}\left(R^{2} \backslash \Gamma\right)$ and $u(x)$ is continuous at the ends of $\Gamma$;

(2) $\nabla u \in C^{0}\left(\overline{R^{2} \backslash \Gamma} \backslash X\right)$, where $X$ is a point set, consisting of the endpoints of $\Gamma$ : $X=\bigcup_{n=1}^{N}\left(x\left(a_{n}\right) \cup x\left(b_{n}\right)\right)$;

(3) in the neighbourhood of any point $x(d) \in X$, for some constants $C>0$ and $\varepsilon>-1$, the inequality

$$
|\nabla u|<\mathcal{C}|x-x(d)|^{\varepsilon}
$$

holds, where $x \rightarrow x(d)$ and $d=a_{n}$ or $d=b_{n}$ for $n=1, \ldots, N$.

Remark 2.1. In the definition of the class $\mathbf{K}$ we consider $\Gamma$ as a set of cracks in a plane. In particular, the notation $C^{0}\left(\overline{R^{2} \backslash \Gamma}\right)$ denotes a class of functions, which are continuously extended on $\Gamma$ from the left and right, but their values on $\Gamma$ from the left and right can be different, so that the functions may have a jump across $\Gamma$.

Let us formulate the jump problem for the harmonic functions in $R^{2} \backslash \Gamma$.

\section{Problem U}

To find a function $u(x)$ of class $\mathbf{K}$, so that $u(x)$ obeys the Laplace equation in $R^{2} \backslash \Gamma$

$$
\Delta u=0, \quad \Delta=\partial_{x_{1}}^{2}+\partial_{x_{2}}^{2}
$$


satisfies the jump boundary conditions

$$
\begin{gathered}
\left.u(x)\right|_{x(s) \in \Gamma^{+}}-\left.u(x)\right|_{x(s) \in \Gamma^{-}}=f_{1}(s), \\
\left.\frac{\partial u}{\partial \mathbf{n}}\right|_{x(s) \in \Gamma^{+}}-\left.\frac{\partial u}{\partial \mathbf{n}}\right|_{x(s) \in \Gamma^{-}}=\left.\beta(s) u(x)\right|_{x(s) \in \Gamma^{+}}+f_{2}(s), \\
\beta(s) \in C^{0, \lambda}(\Gamma), \quad \lambda \in(0,1] ;\left.\quad \beta(s)\right|_{s \in \Gamma} \not \equiv 0,\left.\quad \beta(s)\right|_{s \in \Gamma} \leq 0,
\end{gathered}
$$

and satisfies the following conditions at infinity:

$$
|u(x)|<\mathcal{C}_{1}, \quad|\nabla u(x)|<\mathcal{C}_{2}|x|^{-2},
$$

where $\mathcal{C}_{1}, \mathcal{C}_{2}$ are some constants and $|x|=\sqrt{x_{1}^{2}+x_{2}^{2}} \rightarrow \infty$. Functions $f_{1}(s), f_{2}(s)$ are given on $\Gamma$.

All conditions of the problem must be fulfiled in a classical sense. The case $\beta(s) \equiv 0$ has been studied in [11]. Gradient inequality in (2.5) is not necessary and follows from estimates for harmonic functions. We write this inequality for convenience only.

The problem $\mathbf{U}$ has been formulated in [12]. The goal of the present paper is to obtain new integral representation for the solution of the problem $\mathbf{U}$ and to reduce problem $\mathbf{U}$ to the new integral equation.

Conditions (2.2) at the ends of $\Gamma$ in the formulation of the class $\mathbf{K}$ ensure the absence of point sources at the ends of $\Gamma$. If $\beta(s) \equiv f_{1}(s) \equiv f_{2}(s) \equiv 0$ on $\gamma \subset \Gamma$, then (2.3) holds on $\gamma$ and $u(x)$ is analytic on $\gamma$.

Remark 2.2. Instead of the boundary condition (2.4b) we may consider another boundary condition:

$$
\begin{gathered}
\left.\frac{\partial u}{\partial \mathbf{n}}\right|_{x(s) \in \Gamma^{+}}-\left.\frac{\partial u}{\partial \mathbf{n}}\right|_{x(s) \in \Gamma^{-}}=\left.\beta(s) u(x)\right|_{x(s) \in \Gamma^{-}}+f_{0}(s), \\
\beta(s) \in C^{0, \lambda}(\Gamma), \quad \lambda \in(0,1] ;\left.\quad \beta(s)\right|_{s \in \Gamma} \not \equiv 0,\left.\quad \beta(s)\right|_{s \in \Gamma} \leq 0 .
\end{gathered}
$$

However, this boundary condition can be easily reduced to (2.4b). Indeed, we substitute $\left.u(x)\right|_{x(s) \in \Gamma^{-}}$from (2.4a) to (2.6), then we arrive at $(2.4 \mathrm{~b})$, where

$$
f_{2}(s)=f_{0}(s)-\beta(s) f_{1}(s)
$$

Theorem 2.3. Problem $\mathbf{U}$ has at most one solution.

By $\int_{\Gamma} \cdots$ do one means

$$
\sum_{n=1}^{N} \int_{a_{n}}^{b_{n}} \cdots d \sigma
$$

Now we prove the theorem. The limit values of functions on $\Gamma^{+}$and $\Gamma^{-}$will be denoted by the superscripts "+" and "-", respectively. 
Let $u_{0}(x)$ be a solution of the homogeneous problem $\mathbf{U}$. We will prove that $u_{0}(x) \equiv 0$. To prove this with the help of energy equalities for harmonic functions [13], we envelope open curves by closed contours, tend contours to the curves and use the smoothness of the solution of the problem $\mathbf{U}$. In this way we obtain

$$
\begin{aligned}
\left\|\nabla u_{0}\right\|_{L_{2}\left(C_{r} \backslash \Gamma\right)}^{2} & =\int_{\Gamma} u_{0}^{+}\left(\frac{\partial u_{0}}{\partial \mathbf{n}_{x}}\right)^{+} d s-\int_{\Gamma} u_{0}^{-}\left(\frac{\partial u_{0}}{\partial \mathbf{n}_{x}}\right)^{-} d s+\int_{0}^{2 \pi} u_{0} \frac{\partial u_{0}}{\partial r} r d \varphi \\
& =\int_{\Gamma}\left\{\left(u_{0}^{+}-u_{0}^{-}\right)\left(\frac{\partial u_{0}}{\partial \mathbf{n}_{x}}\right)^{-}+u_{0}^{+}\left[\left(\frac{\partial u_{0}}{\partial \mathbf{n}_{x}}\right)^{+}-\left(\frac{\partial u_{0}}{\partial \mathbf{n}_{x}}\right)^{-}\right]\right\} d s+\int_{0}^{2 \pi} u_{0} \frac{\partial u_{0}}{\partial r} r d \varphi,
\end{aligned}
$$

where $C_{r}$ is the circle of the large radius $r$ with the center in the origin, and $\varphi$ is a polar angle. We suppose that $\Gamma \subset C_{r}$.

Since $u_{0}(x)$ satisfies zero boundary conditions of the homogeneous problem $\mathbf{U}$, we get:

$$
\left\|\nabla u_{0}\right\|_{L_{2}\left(C_{r} \backslash \Gamma\right)}^{2}=-\int_{\Gamma}|\beta(s)|\left|u_{0}^{+}(x(s))\right|^{2} d s+\int_{0}^{2 \pi} u_{0} \frac{\partial u_{0}}{\partial r} r d \varphi
$$

Here we took into account that $\beta(s) \leq 0$ for any $s \in \Gamma$. Putting $r \rightarrow \infty$, we have

$$
\left\|\nabla u_{0}\right\|_{L_{2}\left(R^{2} \backslash \Gamma\right)}^{2}=\lim _{r \rightarrow \infty}\left\|\nabla u_{0}\right\|_{L_{2}\left(C_{r} \backslash \Gamma\right)}^{2}=-\int_{\Gamma}\left|\beta(s) \| u_{0}^{+}(x(s))\right|^{2} d s
$$

because

$$
\lim _{r \rightarrow \infty} \int_{0}^{2 \pi} u_{0} \frac{\partial u_{0}}{\partial r} r d \varphi=0
$$

according to conditions at infinity (2.5). It follows from (2.11) that $\left\|\nabla u_{0}\right\|_{L_{2}\left(R^{2} \backslash \Gamma\right)}^{2}=0$ and, therefore $u_{0}(x) \equiv$ const. Since $\beta(s)$ is continuous on $\Gamma$ and $\beta(s) \not \equiv 0$, there exists $s_{0} \in \Gamma$, so that $\beta\left(s_{0}\right) \neq 0$. Hence, $\beta(s) \neq 0$ in the neighbourhood of $s_{0}$ on $\Gamma$. Thanks to $(2.11), u_{0}^{+}\left(x\left(s_{0}\right)\right)=0$. Consequently, const $=0$ and $u_{0}(x) \equiv 0$ in $R^{2}$ (we used the smoothness of the function $u_{0}(x)$ ensured by the class $\mathbf{K}$ ). Now the statement of the theorem follows from the linearity of the problem $\mathbf{U}$.

\section{The Solution of the Problem}

To construct a solution of the problem $\mathbf{U}$ we impose additional assumptions on the functions $f_{1}(s), f_{2}(s)$ in the boundary conditions $(2.4 \mathrm{a})$ and $(2.4 \mathrm{~b})$ :

$$
\begin{gathered}
f_{1}(s) \in C^{1, \lambda}(\Gamma), \quad f_{2}(s) \in C^{0, \lambda}(\Gamma), \quad \lambda \in(0,1] ; \\
f_{1}\left(a_{n}\right)=f_{1}\left(b_{n}\right)=0, \quad n=1, \ldots, N .
\end{gathered}
$$


The solution of the problem $\mathbf{U}$ can be constructed in the form of a sum of a single layer potential, an angular potential $[4,14]$ and a constant. Set

$$
f_{1}^{\prime}(\sigma)=\frac{d}{d \sigma} f_{1}(\sigma)
$$

and consider a function

$$
u[\mu](x)=v\left[f_{1}^{\prime}\right](x)+w[\mu](x)+\int_{\Gamma} \mu(\sigma) d \sigma,
$$

where

$$
w[\mu](x)=-\frac{1}{2 \pi} \int_{\Gamma} \theta[\mu](\sigma) \ln |x-y(\sigma)| d \sigma
$$

is a single layer potential for (2.3), and

$$
v\left[f_{1}^{\prime}\right](x)=-\frac{1}{2 \pi} \int_{\Gamma} f_{1}^{\prime}(\sigma) V(x, \sigma) d \sigma
$$

is the angular potential $[4,14]$ for $(2.3)$,

$$
\theta[\mu](\sigma)=\mu(\sigma)-\frac{\int_{\Gamma} \mu(\xi) d \xi}{\int_{\Gamma} 1 d \xi} .
$$

We will look for the unknown density $\mu(s)$ in the Hölder space $C^{0, \omega}(\Gamma)$ with some $\omega \in(0,1]$. The kernel $V(x, \sigma)$ is defined (up to indeterminacy $2 \pi m, m= \pm 1, \pm 2, \ldots$ ) by the formulae

$$
\cos V(x, \sigma)=\frac{x_{1}-y_{1}(\sigma)}{|x-y(\sigma)|}, \quad \sin V(x, \sigma)=\frac{x_{2}-y_{2}(\sigma)}{|x-y(\sigma)|}
$$

where

$$
y(\sigma)=\left(y_{1}(\sigma), y_{2}(\sigma)\right) \in \Gamma, \quad|x-y(\sigma)|=\sqrt{\left(x_{1}-y_{1}(\sigma)\right)^{2}+\left(x_{2}-y_{2}(\sigma)\right)^{2}}
$$

One can see that $V(x, \sigma)$ is the angle between the vector $(\overrightarrow{x-y(\sigma)})$ and the direction of the $O x_{1}$ axis. More precisely, $V(x, \sigma)$ is a many-valued harmonic function of $x$ connected with $\ln |x-y(\sigma)|$ by the Cauchy-Riemann relations. Below by $V(x, \sigma)$ we denote an arbitrary fixed branch of this function, which varies continuously with $\sigma$ varying along each curve $\Gamma_{n}(n=$ $1, \ldots, N)$ for given fixed $x \notin \Gamma$. Under this definition of $V(x, \sigma)$, the potential $v\left[f_{1}^{\prime}\right](x)$ is a multivalued function. In order that the potential $v\left[f_{1}^{\prime}\right](x)$ be single-valued, the following additional conditions [10] must hold:

$$
\int_{a_{n}}^{b_{n}} f_{1}^{\prime}(\sigma) d \sigma=f_{1}\left(b_{n}\right)-f\left(a_{n}\right)=0, \quad n=1, \ldots, \mathrm{N} .
$$


Clearly, these conditions are satisfied due to our assumptions (3.1b). Integrating $v\left[f_{1}^{\prime}\right](x)$ by parts and using (3.1b) we express the angular potential in terms of a double-layer potential:

$$
v\left[f_{1}^{\prime}\right](x)=\frac{1}{2 \pi} \int_{\Gamma} f_{1}(\sigma) \frac{\partial}{\partial \mathbf{n}_{y}} \ln |x-y(\sigma)| d \sigma .
$$

Consequently, the angular potential $v\left[f_{1}^{\prime}\right](x)$ satisfies (2.3) outside $\Gamma$ and conditions at infinity (2.5). The single-layer potential $w[\mu](x)$ satisfies conditions (2.5).

It follows from properties of single-layer and angular potentials [4, 13] that the function (3.3) belongs to the class $\mathbf{K}$ and obeys (2.3). Besides, function (3.3) satisfies conditions (2.5) at infinity. Hence, the function (3.3) satisfies all conditions of the problem $\mathrm{U}$ except for the boundary conditions (2.4a) and (2.4b). To satisfy the boundary conditions we, at first, derive the jump formulas for $u(x)$ and its normal derivative on $\Gamma$ using limit properties of single layer potential and angular potential. According to [4], normal derivative of the angular potential $v\left[f_{1}^{\prime}\right](x)$ is continuous across $\Gamma$. The single-layer potential $w[\mu](x)$ is continuous across $\Gamma$ in our assumptions. On the basis of the jump relations on $\Gamma$ for the angular potential and for the normal derivative of the single-layer potential, we obtain [4]

$$
\begin{aligned}
\left.u[\mu](x)\right|_{x(s) \in \Gamma_{n}^{+}}-\left.u[\mu](x)\right|_{x(s) \in \Gamma_{n}^{-}} & =\left.v\left[f_{1}^{\prime}\right](x)\right|_{x(s) \in \Gamma_{n}^{+}}-\left.v\left[f_{1}^{\prime}\right](x)\right|_{x(s) \in \Gamma_{n}^{-}} \\
& =\int_{a_{n}}^{s}\left(\frac{d}{d \sigma} f_{1}(\sigma)\right) d \sigma=f_{1}(s), \quad n=1, \ldots, N, \\
\left.\frac{\partial u[\mu](x)}{\partial \mathbf{n}_{x}}\right|_{x(s) \in \Gamma^{+}}-\left.\frac{\partial u[\mu](x)}{\partial \mathbf{n}_{x}}\right|_{x(s) \in \Gamma^{-}} & =\left.\frac{\partial}{\partial \mathbf{n}_{x}} w[\mu](x)\right|_{x(s) \in \Gamma^{+}} \\
& -\left.\frac{\partial}{\partial \mathbf{n}_{x}} w[\mu](x)\right|_{x(s) \in \Gamma^{-}}=\theta[\mu](s),
\end{aligned}
$$

where conditions $(3.1 \mathrm{~b})$ for $f_{1}(s)$ have been applied. It follows from (3.11) that the function (3.3) satisfies the boundary condition (2.4a). Substituting (3.3) in (2.4b) and using (3.12) and limit formulas for potentials [4], we obtain the integral equation for the function $\mu(s)$ on $\Gamma$

$$
\begin{aligned}
& \mu(s)+\beta(s) \frac{1}{2 \pi} \int_{\Gamma} \theta[\mu](\sigma) \ln |x(s)-y(\sigma)| d \sigma \\
& -\left(\beta(s)+\frac{1}{\int_{\Gamma} 1 d \xi}\right) \int_{\Gamma} \mu(\sigma) d \sigma=F(s), \quad s \in \Gamma,
\end{aligned}
$$

where

$$
F(s)=f_{2}(s)+\beta(s)\left[\frac{1}{2} f_{1}(s)+\frac{1}{2 \pi} \int_{\Gamma} f_{1}(\sigma) \frac{\partial \ln |x(s)-y(\sigma)|}{\partial \mathbf{n}_{y}} d \sigma\right],
$$

and conditions $(3.1 \mathrm{~b})$ have been used. The term in the square brackets is the limit value of a double layer potential (3.10) on $\Gamma^{+}$. The last term in square brackets is the direct value of the double layer potential (3.10) on $\Gamma$. This term belongs to $C^{0, \lambda}(\Gamma)$ in $s$ [see Lemma 1 in [5] and 
Lemma 2 in [4]] if conditions (3.1a) hold. Taking into account conditions (3.1a) we observe that $F(s) \in C^{0, \lambda}(\Gamma)$. The kernel of the integral term in (3.13) has logarithmic singularity if $s=\sigma$. It follows from [Lemma 2 in [15]] that the integral operator in (3.13):

$$
\mathbf{A} \mu=\frac{1}{2 \pi} \int_{\Gamma} \theta[\mu](\sigma) \beta(s) \ln |x(s)-y(\sigma)| d \sigma
$$

is a compact operator mapping $C^{0}(\Gamma)$ into itself. Moreover, this integral operator maps $C^{0}(\Gamma)$ into $C^{0, \omega}(\Gamma)$ with $\omega=\min \{\lambda, 1 / 4\}$. Hence if $\mu(s)$ is a solution of (3.13) in $C^{0}(\Gamma)$, then proceeding from identity (3.13) for $\mu(s)$ we observe that $\mu(s)$ automatically belongs to $C^{0, \omega}(\Gamma)$, where $\omega=\min \{\lambda, 1 / 4\}$. Consequently, if $\mu(s) \in C^{0}(\Gamma)$ is a solution of (3.13), then according to properties of potentials $[4,11,13]$ the function (3.3) belongs to the class $\mathbf{K}$ and satisfies all conditions of the problem $\mathbf{U}$.

We arrive at the following lemma.

Lemma 3.1. (1) If $F(s) \in C^{0, \lambda}(\Gamma), \lambda \in(0,1]$, then any solution of (3.13) in $C^{0}(\Gamma)$ automatically belongs to $C^{0, \omega}(\Gamma)$ with $\omega=\min \{\lambda, 1 / 4\}$.

(2) Let conditions (3.1a) and (3.1b) hold. If $\mu(s)$ is a solution of (3.13) in $C^{0}(\Gamma)$, where $F(s)$ is given by (3.14), then the function (3.3) is a solution of the problem $\boldsymbol{U}$.

Remark 3.2. Notice that in [12] the integral representation for the solution to the problem $\mathbf{U}$ has been obtained in the form

$$
\begin{aligned}
u[\mu](x)= & v\left[f_{1}^{\prime}\right](x) \\
& -\frac{1}{2 \pi} \int_{\Gamma} \mu(\sigma) \ln |x-y(\sigma)| d \sigma+\frac{(1 / 2 \pi) \int_{\Gamma} \mu(\sigma) \int_{\Gamma} \beta(s) \ln |x(s)-y(\sigma)| d s d \sigma}{\int_{\Gamma} \beta(s) d s} \\
& -\frac{\int_{\Gamma}\left(\beta(s)\left[(1 / 2) f_{1}(s)+(1 / 2 \pi) \int_{\Gamma} f_{1}(\sigma)\left(\left(\partial / \partial \mathbf{n}_{y}\right) \ln |x(s)-y(\sigma)| d \sigma\right)\right]+f_{2}(s)\right) d s}{\int_{\Gamma} \beta(s) d s} .
\end{aligned}
$$

This function a priori does not satisfy conditions at infinity (2.5) for an arbitrary $\mu$ unlike the function (3.3). However being substituted into the boundary condition (2.4b) this integral representation for a solution leads to such an integral equation for the unknown function $\mu$ (see [12]) that this integral equation satisfies two important properties. First, any solution $\mu$ of the integral equation in [12] falls in the class of functions that ensure satisfaction of conditions at infinity for the solution of the boundary value problem. Second, the integral equation in [12] is uniquely solvable. In fact, the integral representation for a solution of the problem $\mathbf{U}$ has been taken in [12] in the above form in order to obtaining integral equation satisfying two aforementioned properties (see details and proofs in [12]). However, the integral representation for a solution of the problem $\mathbf{U}$ given by (3.3) is much simpler than the integral representation for a solution of the problem $\mathbf{U}$ suggested in [12] and written out above. Finding simple integral representation for a solution is very important for further numerical treatment of the problem by the boundary integral equation method. 
Below we look for a solution of (3.13) in $C^{0}(\Gamma)$ owing to Lemma 3.1. The operator

$$
\mathbf{B} \mu=\left(\beta(s)+\frac{1}{\int_{\Gamma} 1 d \xi}\right) \int_{\Gamma} \mu(\sigma) d \sigma
$$

is finite dimensional operator mapping $C^{0}(\Gamma)$ into itself, and so it is compact [16]. Consequently, the integral operator $(\mathbf{A}-\mathbf{B}) \mu$ in (3.13) is a compact operator mapping $C^{0}(\Gamma)$ into itself. Therefore (3.13) is a Fredholm integral equation of the second kind and index zero in $C^{0}(\Gamma)$, that is, $(3.13)$ is subject to the Fredholm alternative in $C^{0}(\Gamma)$ (see $\left.[16,17]\right)$.

Let us show that the homogeneous equation (3.13) has only a trivial solution in $C^{0}(\Gamma)$. Let $\mu^{0}(s)$ be a solution of the homogeneous equation (3.13). According to lemma, $\mu^{0}(s)$ automatically belongs to $C^{0, \omega}(\Gamma)$. It follows from point 2 of Lemma 3.1 that

$$
\begin{aligned}
u\left[\mu^{0}\right](x) & =w\left[\mu^{0}\right](x)+\int_{\Gamma} \mu^{0}(\sigma) d \sigma \\
& =-\frac{1}{2 \pi} \int_{\Gamma} \theta\left[\mu^{0}\right](s) \ln |x(s)-y(\sigma)| d \sigma+\int_{\Gamma} \mu^{0}(\sigma) d \sigma
\end{aligned}
$$

is a solution of the homogeneous problem U. Taking into account Theorem 2.3, we observe that

$$
u\left[\mu^{0}\right](x) \equiv 0, \quad x \in R^{2} \backslash \Gamma,
$$

since the homogeneous problem $\mathbf{U}$ has only a trivial solution. Using the jump relations for the normal derivative of the single layer potential on $\Gamma$, we obtain

$$
\begin{aligned}
\left.\frac{\partial u\left[\mu^{0}\right](x)}{\partial \mathbf{n}_{x}}\right|_{x \in \Gamma^{+}}-\left.\frac{\partial u\left[\mu^{0}\right](x)}{\partial \mathbf{n}_{x}}\right|_{x \in \Gamma^{-}} & =\theta\left[\mu^{0}\right](s) \\
& =\mu^{0}(s)-\frac{\int_{\Gamma} \mu^{0}(\xi) d \xi}{\int_{\Gamma} 1 d \xi} \equiv 0, \quad s \in \Gamma .
\end{aligned}
$$

Consequenly,

$$
\mu^{0}(s)=\frac{\int_{\Gamma} \mu^{0}(\xi) d \xi}{\int_{\Gamma} 1 d \xi}, \quad s \in \Gamma .
$$

Substituting (3.18) into (3.19) we have

$$
\int_{\Gamma} \mu^{0}(\xi) d \xi=0
$$


It follows from (3.21) that

$$
\mu^{0}(s) \equiv 0, \quad s \in \Gamma .
$$

Thus, we have proved that the homogeneous equation (3.13) has only a trivial solution. According to Fredholm alternative, the inhomogeneous equation (3.13) is uniquely solvable in $C^{0}(\Gamma)$ for any $F(s) \in C^{0}(\Gamma)$.

Theorem 3.3. Equation (3.13) has unique solution $\mu(s) \in C^{0}(\Gamma)$ for any $F(s) \in C^{0}(\Gamma)$. In addition, if $F(s) \in C^{0, \lambda}(\Gamma), \lambda \in(0,1]$, then the unique solution of $(3.13)$ in $C^{0}(\Gamma)$ belongs to $C^{0, \omega}(\Gamma)$ with $\omega=\min \{\lambda, 1 / 4\}$.

The latter statement of Theorem 3.3 follows from Lemma 3.1. As aforementioned, if conditions (3.1a) and (3.1b) hold, then $F(s) \in C^{0, \lambda}(\Gamma), \lambda \in(0,1]$. Using Lemma 3.1 we obtain solvability of the problem $\mathbf{U}$.

Theorem 3.4. If conditions (3.1a) and (3.1b) hold, then the solution of the problem $\boldsymbol{U}$ exists and is given by (3.3), where $\mu(s) \in C^{0, \omega}(\Gamma)(\omega=\min \{\lambda, 1 / 4\})$ is a solution of (3.13) in $C^{0}(\Gamma)$ ensured by Theorem 3.3.

It follows from [Theorem 5 in [4]] that the condition (2.2) for $|\nabla u|$ is fulfied for any $\varepsilon \in$ $(0,1)$, that is, for any small positive $\varepsilon$. In other words, $\nabla u(x)$ does not have power singularity at the ends of $\Gamma$. It will be shown in next section that $\nabla u$ has logarithmic singularity or, in certain cases, does not have singularity at all. Explicit formulas for singularities of $\nabla u$ at the ends of $\Gamma$ will be presented and discussed in the next section.

\section{Singularities of a Gradient of a Solution at the Ends of $\Gamma$}

In this section by $u(x)$ we denote the solution of the problem $\mathrm{U}$ ensured by the Theorem 3.4. According to (2.2), $\nabla u$ may be unbounded at the ends of $\Gamma$. The explicit expressions for singularities of $\nabla u$ can be obtained from the formulas for singularities of derivatives of single layer and angular potentials near edges $[4,5]$. Let $x(d)$ be one of the end-points of $\Gamma$. In the neighbourhood of $x(d)$ we introduce the system of polar coordinates

$$
x_{1}=x_{1}(d)+|x-x(d)| \cos \varphi, \quad x_{2}=x_{2}(d)+|x-x(d)| \sin \varphi .
$$

We will assume that $\varphi \in(\alpha(d), \alpha(d)+2 \pi)$ if $d=a_{n}$ and $\varphi \in(\alpha(d)-\pi, \alpha(d)+\pi)$ if $d=b_{n}$ for $n=1, \ldots, N$. Recall that $\alpha(s)$ is the angle between the tangent vector $\tau_{x}$ to $\Gamma$ at the point $x(s)$ and the direction of the $O x_{1}$ axis. Hence, $\alpha(d)=\alpha\left(a_{n}+0\right)$ if $d=a_{n}$ and $\alpha(d)=\alpha\left(b_{n}-0\right)$ if $d=b_{n}$. Consequently the angle $\varphi$ varies continuously in the neighbourhood of the point $x(d)$, cut along the contour $\Gamma$.

Recall that $X$ is a set of end-points of $\Gamma$. Computing singularities of $\nabla u$ in the same way as in $[4,5]$ we arrive to the following assertion. 
Theorem 4.1. Let $x \rightarrow x(d) \in X$. Then in the neigbourhood of the point $x(d)$ the derivatives of the solution of the problem $\boldsymbol{U}$ have the following behaviour:

$$
\begin{aligned}
\frac{\partial}{\partial x_{1}} u(x)= & -(-1)^{m} \frac{f_{1}^{\prime}(d)}{2 \pi}[-\sin \alpha(d) \ln |x-x(d)|+\varphi \cos \alpha(d)] \\
& -(-1)^{m} \frac{\theta[\mu](d)}{2 \pi}[\cos \alpha(d) \ln |x-x(d)|+\varphi \sin \alpha(d)]+O(1), \\
\frac{\partial}{\partial x_{2}} u(x)= & -(-1)^{m} \frac{f_{1}^{\prime}(d)}{2 \pi}[\cos \alpha(d) \ln |x-x(d)|+\varphi \sin \alpha(d)] \\
& +(-1)^{m} \frac{\theta[\mu](d)}{2 \pi}[-\sin \alpha(d) \ln |x-x(d)|+\varphi \cos \alpha(d)]+O(1),
\end{aligned}
$$

where $m=0$ if $d=a_{n}$ and $m=1$ if $d=b_{n}$ for $n=1, \ldots, N$.

Remark 4.2. By $O(1)$ we denote functions which are continuous at the point $x(d)$. Furthermore, the functions denoted by $O(1)$ are continuous in the neighbourhood of the point $x(d)$, cut along the contour $\Gamma$.

According to Theorem 4.1, $\nabla u$ has logarithmic singularities at the ends of cracks $\Gamma$ in general. However, if $f_{1}^{\prime}(d)=\theta[\mu](d)=0$ at the end $x(d) \in X$, then there is no any singularity of $\nabla u$ at the end $x(d)$. Moreover, $\nabla u$ is continuous at this end. If $f_{1}^{\prime}(d) \neq 0$ or $\theta[\mu](d) \neq 0$, then $\nabla u$ has a logarithmic singularity at $x(d) \in X$.

Let us compare our results with singularities of a solution gradient in the Dirichlet and Neumann problems at the exterior of cracks in a plane. In these problems either Dirichlet or Neumann boundary condition has been specified on the cracks instead of (2.4a) and (2.4b). It was shown in $[4,5]$ that the solution gradient in the Dirichlet and Neumann problems in general tends at infinity as $O\left(|x-x(d)|^{-1 / 2}\right)$ when $x \rightarrow x(d) \in X$. According to Theorem 4.1, the edge singularities of $\nabla u$ in the jump problem are generally logarithmic. Thus, the jump problem and Dirichlet (or Neumann) problem have as a rule different orders of singularities at the ends of cracks, so that the singularities in the jump problem are weaker. We can conclude that the behaviour of the solution in the jump problem is essentially different from behaviour of the solution in the Dirichlet (or Neumann) problem. The discussed properties of singularities may be effectively used to select adequate model describing cracked media or media with membranes.

\section{Comparison of Results and Conclusions}

Let us discuss obtained results and compare them with previous treatments of the problem $\mathbf{U}$.

Notice that the case $\beta \equiv 0$ in $(2.4 b)$ is excluded from consideration. This case has been studied in [11]. It is curious that the problem $\mathbf{U}$ is not uniquely solvable in this case under usual conditions at infinity. There exists solvability condition, and if it holds, then the problem $\mathrm{U}$ has infinitely many solutions that differ by additive constant as in Neumann problem. From the other hand this case admits explicit solution, so that solving of integral equation is not needed.

For the first time the problem $\mathbf{U}$ with $\beta \not \equiv 0$ has been treated in [12]. To reduce the problem to the uniquely solvable integral equation, very special and unusual technique has 
been applied. The solution was looked in the form of single-layer and angular potentials plus some functionals depending on density $\mu$ in a single-layer potential and on boundary data. The solution was looked for in the form that a priori does not satisfy conditions at infinity. However, being substituted into the boundary condition, the solution in the form of potentials and additional functionals produces such an integral equation, that any solution $\mu$ of the integral equation automatically falls in the class of functions that ensure satisfaction of conditions at infinity for the solution of the boundary value problem. More precisely, additional functionals of $\mu$ and of boundary data in the form of a solution have been chosen $a$ priori in such a way to obtain this special property of solutions of the integral equation. Moreover, these additional functionals of $\mu$ and of boundary data have been chosen in such a way to obtain uniquely solvable integral equation at the same time. The only problem with approach in [12] was concluded in the fact that the final expression for the solution of the boundary value problem appeared to be rather complicated. In the present paper we derived another integral representation for a solution of problem $\mathbf{U}$ and we have reduced problem $\mathbf{U}$ to another uniquely solvable integral equation. The idea of new approach is the following. We a priori choose such a representation for a solution of the problem $\mathbf{U}$ in the form of potentials that this chosen form satisfy two properties. First, it automatically satisfies conditions at infinity for any density $\mu$ in the single-layer potential. Second, it reduces the problem to the uniquely solvable integral equation. It should be stressed that both integral equation and integral representation for a solution obtained in the present paper are completely different from those obtained in [12] and are essentially simpler. Finding simple integral representation for a solution and finding simple uniquely solvable integral equation are very important for further numerical treatment of the problem by the boundary integral equation method.

\section{Acknowledgment}

The research was supported by JSPS and this support is gratefully acknowledged.

\section{References}

[1] P. Wolfe, "An existence theorem for the reduced wave equation," Proceedings of the American Mathematical Society, vol. 21, pp. 663-666, 1969.

[2] M. Durand, "Layer potentials and boundary value problems for the Helmholtz equation in the complement of a thin obstacle," Mathematical Methods in the Applied Sciences, vol. 11, pp. 185-213, 1989.

[3] I. K. Lifanov, Singular Integral Equations and Discrete Vortices, VSP, Utrecht, The Netherlands, 1996.

[4] P. A. Krutitskiı̌, "The Dirichlet problem for the Helmholtz equation in the exterior of cuts in the plane," Computational Mathematics and Mathematical Physics, vol. 34, pp. 1073-1090, 1994.

[5] P. A. Krutitskir, "The Neumann problem for the Helmholtz equation in the exterior of cuts in the plane," Computational Mathematics and Mathematical Physics, vol. 34, pp. 1421-1431, 1994.

[6] P. A. Krutitskii, "The Dirichlet problem for the two-dimensional Laplace equation in a multiply connected domain with cuts," Proceedings of the Edinburgh Mathematical Society. Series II, vol. 43, no. 2, pp. 325-341, 2000.

[7] P. A. Krutitskii, "The Neumann problem in a 2-D exterior domain with cuts and singularities at the tips," Journal of Differential Equations, vol. 176, no. 1, pp. 269-289, 2001.

[8] P. A. Krutitskii, "Wave propagation in a 2D external domain bounded by closed and open curves," Nonlinear Analysis. Theory, Methods \& Applications, vol. 32, no. 1, pp. 135-144, 1998.

[9] P. A. Krutitskii, "The Neumann problem on wave propagation in a 2-D external domain with cuts," Journal of Mathematics of Kyoto University, vol. 38, no. 3, pp. 439-451, 1998.

[10] N. I. Muskhelishvili, Singular Integral Equations, Wolters-Noordhoff Publishing, Groningen, The Netherlands, 1972. 
[11] P. A. Krutitskii, "Explicit solution of the jump problem for the Laplace equation and singularities at the edges," Mathematical Problems in Engineering, vol. 7, no. 1, pp. 1-13, 2001.

[12] P. A. Krutitskii, "The modified jump problem for the Laplace equation and singularities at the tips," Journal of Computational and Applied Mathematics, vol. 183, no. 1, pp. 232-240, 2005.

[13] V. S. Vladimirov, Equations of Mathematical Physics, vol. 3, Marcel Dekker, New York, NY, USA, 1971.

[14] S. A. Gabov, "An angular potential and its applications," Mathematics of the USSR, Sbornik, vol. 32, pp. 423-436, 1977.

[15] P. A. Krutitskiı̌, "A boundary value problem for the Laplace equation outside cuts on the plane with different conditions of the third kind on opposite sides of the cuts," Differential Equations, vol. 45, no. 1, pp. 86-100, 2009.

[16] S. G. Krĕnn, Ed., Functional Analysis, Wolters-Noordhoff Publishing, Groningen, The Netherlands, 1972.

[17] L. V. Kantorovich and G. P. Akilov, Functional Analysis, Pergamon Press, Oxford, UK, 2nd edition, 1982. 


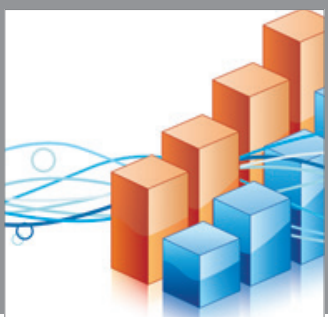

Advances in

Operations Research

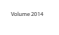

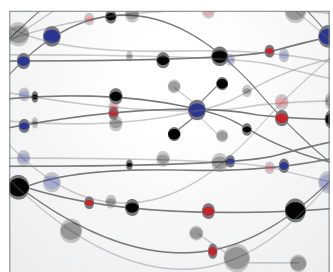

\section{The Scientific} World Journal
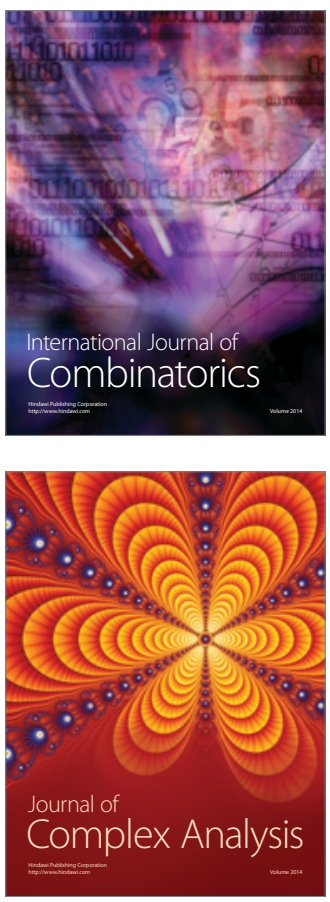

International Journal of

Mathematics and

Mathematical

Sciences
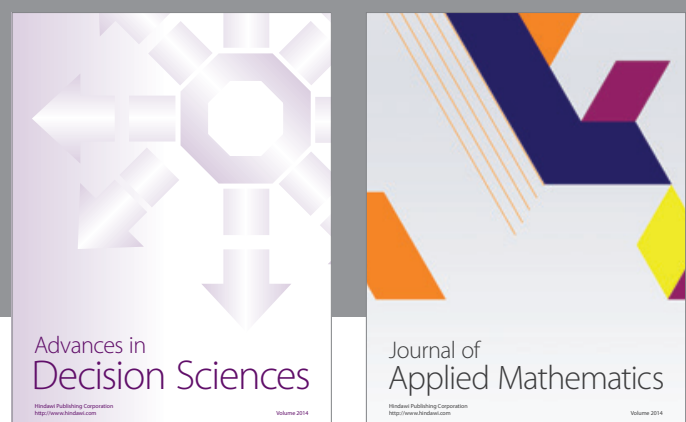

Journal of

Applied Mathematics
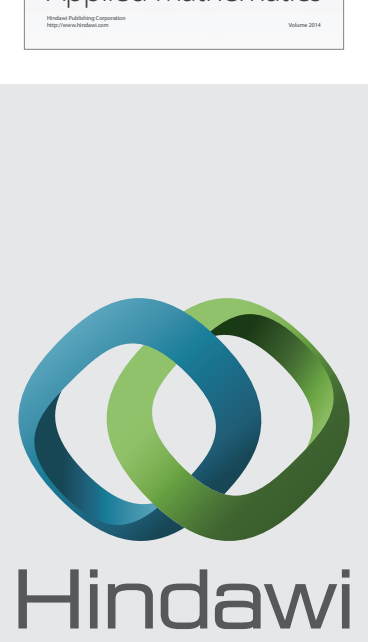

Submit your manuscripts at http://www.hindawi.com
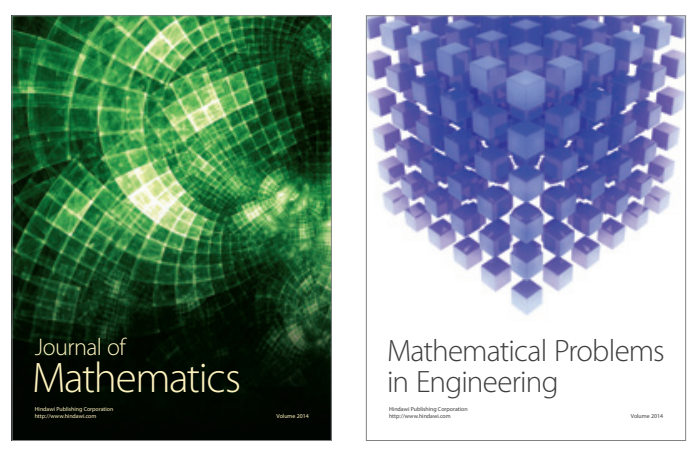

Mathematical Problems in Engineering
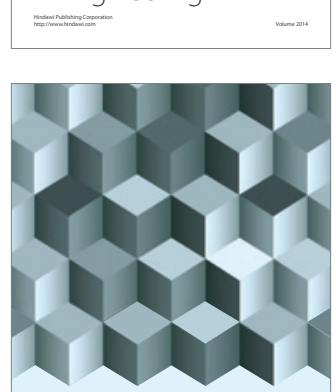

Journal of

Function Spaces
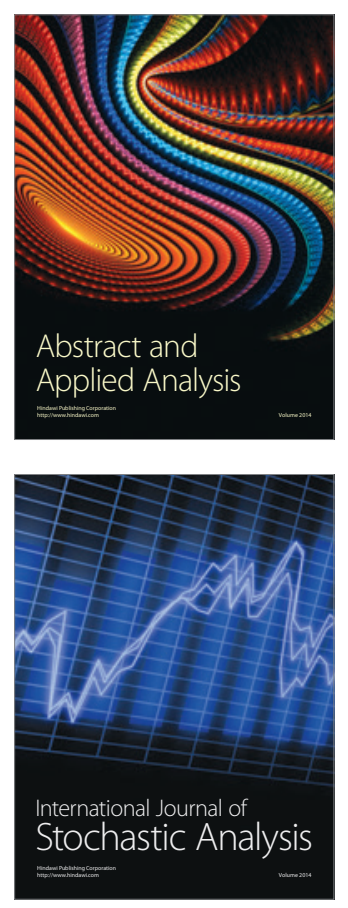

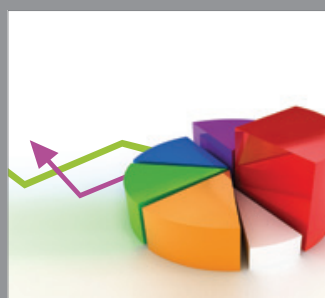

ournal of

Probability and Statistics

Promensencen
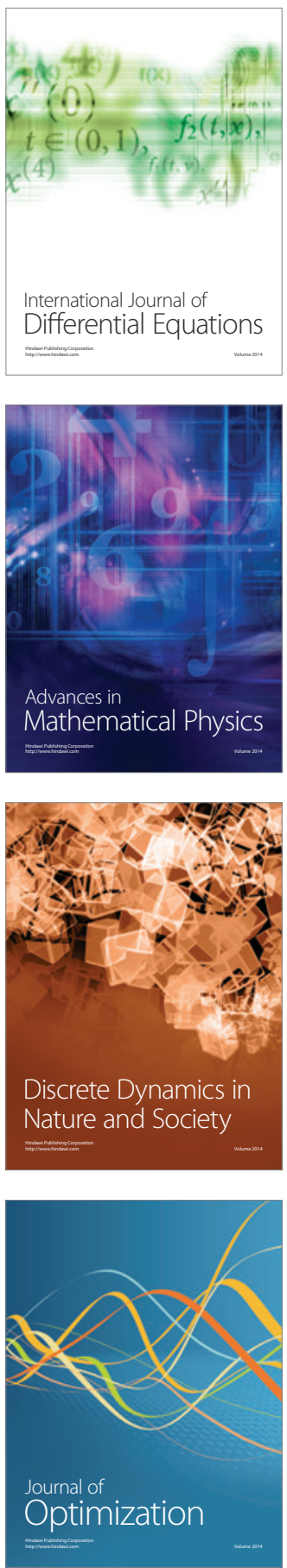